\title{
Environmental Field Estimation with Hybrid-Mobility Sensor Networks
}

\author{
William C. Evans ${ }^{1,2}$, Duarte Dias ${ }^{1}$ and Steven Roelofsen ${ }^{1}$ and Alcherio Martinoli ${ }^{1}$
}

\begin{abstract}
The remarkable accessibility of modern flying robots makes them an attractive platform for environmental sensing. However, low cost and ease of use are currently incompatible with large payloads, severely limiting the choice of sensor and ultimately modality. This paper describes the design of a system for using a small infrared thermometer to estimate the surface temperature over an area that is large compared to the area measured by the sensor, by mounting it on a flying robot. We leverage a priori knowledge about the spatial statistics of the phenomena under measure in order to plan an informative sampling path, fusing observations by Gaussian process regression. Our approach is designed to be evaluated in an indoor testbed, in which a quadrotor, in cooperation with simulated static sensing nodes, estimates the spatial distribution of surface temperature over a controlled thermal gradient. We perform extensive systematic experimentation both in simulation and our real-world testbed environment, with our algorithm estimating surface temperature to an accuracy of up to $2.1{ }^{\circ} \mathrm{C}$ over a $16 \mathrm{~m}^{2}$ area ranging in value from $25-65^{\circ} \mathrm{C}$.
\end{abstract}

\section{INTRODUCTION}

The remarkable accessibility of modern flying robots makes them an attractive platform for environmental sensing. Quadrotor aircraft in particular have seen a recent surge in consumer popularity due in large part to their robustness, stability and price. Autonomous and semi-autonomous fixedwing aircraft have seen increasing use in environmental sensing and mapping, with the rise of companies such as senseFly [1]. Indeed, mobile environmental sensing offers to ameliorate a common issue in static sensor deploymentslimited coverage.

However, this approach is not without its drawbacks. Commercially available autonomous flying platforms are still very limited in terms of flight time, and must be launched and/or landed manually. Furthermore, at the time of this publication, most areas of the developed world require that autonomous aircraft be supervised at all times by a human operator. Ultimately, the combination of these issues suggests that while mobile platforms offer a means of sensing with high spatial density, they suffer from limited overall autonomy, thus limiting the frequency with which they might collect data.

We thus conclude that current technology requires using static and mobile sensing nodes in tandem to achieve high measurement density and accuracy in both time and space.

\footnotetext{
${ }^{1}$ Distributed Intelligent Systems and Algorithms Laboratory; School of Architecture, Civil and Environmental Engineering; École Polytechnique Fédérale de Lausanne; 1015 Lausanne, Switzerland \{william.evans, duarte.dias, steven.roelofsen, alcherio.martinoli\}eepfl.ch

${ }^{2}$ Google; 1600 Amphitheatre Parkway, Mountain View, CA 94043, USA wevans@google.com
}

In this paper we construct an approach to integrating a mobile sensor node with a static network, leveraging existing infrastructure to guide its data collection to best complement measurements from static nodes.

One must also consider the method for deciding where to make observations in attempting to best improve their estimation of a particular field. We implement and evaluate approaches both for sensor placement, i.e., where best to place static sensors, and path planning, i.e., how a mobile sensor should choose to move through the space, and ultimately combine the two to form a heterogeneous sensor network.

Under our proposed approach, we leverage an a priori statistical estimate of the spatial structure of the quantity under measure in order to plan our next observation. We implement our algorithms on a real-world flying robot, equipped with an infrared thermometer, and assess their performance both in simulation and a novel indoor testbed.

In order to evaluate our approach, we propose the use of electrical heaters to create a repeatable thermal gradient. The heaters are placed with uniformly random position and orientation inside the area of flight, producing a field that can be statistically characterized. Ground truth is provided using a high-resolution thermal camera, and is compared to a flying robot's estimation produced by scanning the area with an infrared thermometer.

The rest of this paper is structured as follows. In Section II we discuss related work in sensor placement, path planning and estimation. In Section III, we describe our experimental platform, including the sensor and mobile node. In Section IV, we describe our technical approach, including our sensing model, and sensing and estimation algorithms. In Section V, we proceed to describe the tools used to evaluate our approach, i.e., our simulation and real-world experimental setup. In Section VI, we present our experimental results, and finally offer our conclusions in Section VII.

\section{RELATED WORK}

Environmental sensor placement and control is an active area of research. Assuming that the field under measure can be modeled as a Gaussian process (GP), researchers have proposed multiple criteria for predicting the "informativeness" of a sampling position, most commonly either the variance of the GP or mutual information. Mutual information has been shown to be a more effective measure of sample utility [2], and has subsequently been used for both sensor placement [3] and the control of mobile sensors [4], [5], [6]. Alternative methods have been proposed to better cope with a lack a priori information about the field under measure. Ouyang et al. propose a tradeoff between gathering data that 


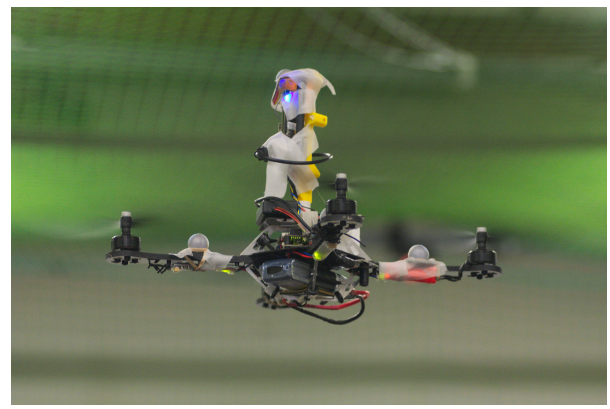

Fig. 1: the AscTec Hummingbird. We have mounted a vertical tower on the Hummingbird with a Gumstix Overo Airstorm, which is used for general computation, communication, and sensor data collection. Infrared-reflective spheres are used for indoor localization.

improves estimation accuracy versus data that better reveals the correlation structure of the process [7].

Some of the works listed above use more nuanced statistical models and target more complex fields (e.g., anisotropic, nonstationary) than the approaches we develop in this paper; however, to the best of our knowledge, none of them have been systematically validated via real experiments. Our goal in this paper is specifically to develop an approach that lends itself well to real-world experimentation on a robotic platform. We thus evaluate our approaches exclusively on homogeneous random fields, so that we might build a baseline understanding of the performance of these approaches on a real system.

\section{MATERIALS}

Mobile sampling requires the integration of a number of technologies. In this section we describe and motivate our choices for each piece of our platform, from the quadrotorbased mobile node to its sensor loadout.

\section{A. Mobile Platform}

Our mobile platform is built around the Ascending Technologies Hummingbird quadrotor [8]. The Hummingbird is a small, commercially available aircraft equipped with four brushless motors for flight (see Figure 1). It weighs approximately $510 \mathrm{~g}$ with battery out of the box, has a flight time of 15-20 minutes, and a maximum airspeed of $15 \mathrm{~m} / \mathrm{s}$. It is capable of carrying a payload of up to $200 \mathrm{~g}$.

The Hummingbird benefits from Markus W. Achtelik's asctec_mav_framework package [9], which allows it to interface with any computing hardware that supports the Robot Operating System (ROS) [10]. ROS is a widelyused robotics middleware that primarily provides hardware abstraction and message-passing based composition of various software components, greatly easing its integration into our indoor flying arena (see Section V).

\section{B. Sensing Payload}

The Phidgets 1045 is an infrared temperature sensor [11] that provides scalar surface temperature measurements at

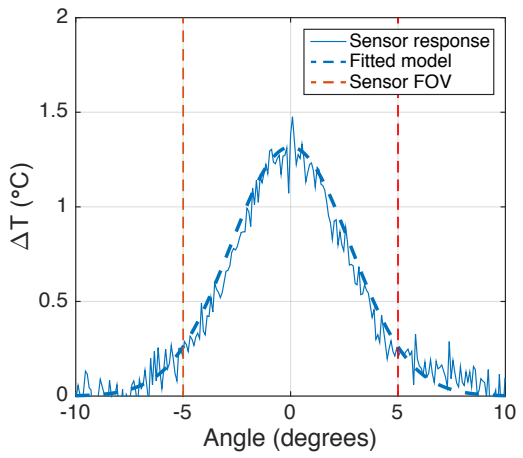

Fig. 2: Sensor response while downward-facing and swept over a heat source at $1.62 \mathrm{~m}$ height. The $\mathrm{x}$-axis gives the angle between the sensor and the heat source. The vertical red lines represent the sensor's FOV as stated in its datasheet.

$31 \mathrm{~Hz}$. Interfaced with the onboard Gumstix via USB, it has a $10^{\circ} \mathrm{FOV}$ and a listed error of $\pm 2{ }^{\circ} \mathrm{C}$.

In order to characterize the sensor, we mounted it on a 3-axis robot alongside a thermal camera. We performed a systematic perpendicular scan over a small heat source and measured the Phidgets 1045's response as a function of the angle between the sensor and the source. We found that the sensor's response - in remote sensors such the Phidgets 1045, this is often referred to as the point spread function (PSF)could be modeled as a Gaussian weighted average over the area covered by the sensor (see Figure 2). This matches published literature on PSFs for electro-optical sensors [12].

\section{METHODS}

\section{A. Spatial Model}

Gaussian processes are commonly used to represent spatially varying processes, and form the underpinnings of Kriging, a widely used interpolation technique in environmental science.

1) Gaussian Processes: A stochastic process $\mathrm{X}$ is a Gaussian process (GP) if and only if for every member of some index set $i \in \Lambda, \mathbf{X}_{i}$ is a Gaussian random variable, and further, for every $k$-length $\lambda \subseteq \Lambda$ with finite $k, \mathbf{X}_{\lambda_{1}, \ldots, \lambda_{k}}$ is a multivariate Gaussian random variable.

In this paper, we use GPs to model a spatially varying environmental process on a plane. We model this process by discretizing it into a grid of points, $p_{i}$, for each $i \in \Lambda$. That is, each $\mathbf{X}_{i}$ can be mapped to a point on a grid, with position $p_{i}$.

2) Homogeneity: GPs have many desirable properties that result from their underlying normal distribution, e.g. they are straightforward to manipulate and are computationally tractable, as we will see in the rest of this paper. Toward this end, we will restrict our model even further, to only consider GPs which are both stationary and isotropic.

Any GP is completely described by its mean function $\mu$, and covariance function $K$, the latter of which is sometimes called its kernel. Given $\mathbf{X} \sim \operatorname{GP}(\mu, K), \mathbf{X}$ is considered to be stationary if and only if $\mu$ is constant inside $\Lambda$, and 


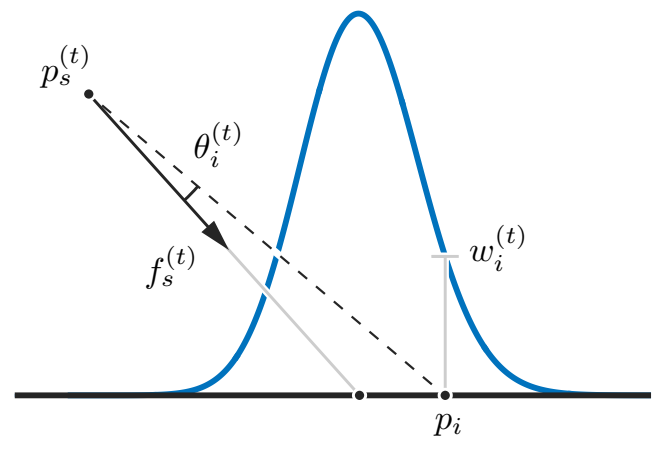

Fig. 3: Sensing model. Consider the sensor to be at $p_{s}^{(t)}$ for some time $t$. The value returned by the sensor is modeled by the truncated Gaussian-weighted sum of field values at discrete points inside its field of view. Point $\mathbf{X}_{i}$ is given a weight $w_{i}^{(t)}$ depending on its angle $\theta_{i}^{(t)}$ to the direction the sensor is facing, $f_{s}^{(t)}$.

critically, if $K_{i j}$ is solely a function of the vector $p_{i}-p_{j}$. X is furthermore isotropic if and only if $K$ depends only on the distance between points $p_{i}$ and $p_{j}, d_{i j}$. A GP that is both stationary and isotropic is called homogeneous.

\section{B. Sensing Model}

Robotic platforms offer a particular advantage for measuring certain environmental quantities with unprecedented spatial and temporal resolution. Previously, common options for remote sensing were either the use of relatively low resolution satellite data, or hiring a manned aircraft, e.g., as in the Léman-Baïkal survey [13]. Even when remote data is available for a field of interest, such as surface temperature (e.g., via satellite) it may be of insufficient resolution (in both time and space) to for example drive detailed models of microclimatic phenomena. Flying robots offer the ability to take in situ measurements with a sensor of choice with high spatial density.

However, inexpensive flying robots can currently carry only a modest payload, and many useful sensors are simply too heavy to be mounted (e.g., high-resolution hyperspectral cameras [14], [15] or thermal cameras [15], [16]). In this paper we develop an approach for using a low resolution (i.e., single pixel) thermal sensor to approximate the field measured by a high-quality thermal camera. Our algorithm exploits a priori knowledge of a field's statistical structure in order to rapidly build an accurate estimation. In uncontrolled environments (i.e., field deployments), one must make an approximation of such structure, either based on data collected under similar conditions, simulation, expert knowledge, and/or an already deployed static network that the mobile sensor is intended to complement. This structure, along with a model of the thermal sensor's response, is used to perform Gaussian process regression after each measurement.

The sensors we consider in our work operate as single pixel cameras, and can be modeled by a Gaussian weighted average with the angle between a particular point on the target surface and the sensor's orientation (see Figure 3). Thus, at time $t$ the weight a given point $\mathbf{X}_{i}$ contributes to the sensor's observation is:

$$
w_{i}^{(t)}= \begin{cases}\mathcal{N}\left(\theta_{i}^{(t)}, 0, \sigma_{s}\right) & \text { if } \theta_{i}^{(t)} \leq \theta_{s} \\ 0 & \text { otherwise }\end{cases}
$$

with FOV $2 \theta_{s}$ and standard deviation $\sigma_{s} . \theta_{i}^{(t)}$ is the angle between the facing direction of the sensor, $f_{s}^{(t)}$, and the vector between the position of the sensor, $p_{s}^{(t)}$, and the position of the respective element of the GP, $p_{i}$ :

$$
\theta_{i}^{(t)}=\cos ^{-1} \frac{f_{s}^{(t)} \cdot\left(p_{s}^{(t)}-p_{i}\right)}{\left|f_{s}^{(t)}\right| \cdot\left|\left(p_{s}^{(t)}-p_{i}\right)\right|}
$$

Under this model, the sensor sees a low-pass filtered picture of the underlying field, where the width of the blur increases with sensor height. Let $x$ denote the instantiation of some GP $\mathbf{X}$. The value returned by the sensor is thus:

$$
v_{s}^{(t)}=\sum_{i}^{\Lambda} w_{i}^{(t)} x_{i}+\epsilon
$$

for some Gaussian noise $\epsilon \sim \mathcal{N}\left(0, \sigma_{n}^{2}\right)$. Note that a weighted sum of Gaussians with same mean and covariance is also Gaussian.

\section{Incorporating Observations}

We use Gaussian process regression (GPR) to fuse observations from our sensor into an estimate of the underlying field. GPR is a well-studied technique that has benefited from popularity in the machine learning community-and as such, is reasonably performant, even for a large number of states.

We can derive the conditioned GP by standard Bayesian methods, which yield the following equations for our posterior mean vector and covariance matrix, given observations from time $t_{1}, \ldots, t_{k}$ :

$$
\begin{aligned}
& \mathbb{E} {\left[\mathbf{X} \mid v_{s}^{\left(t_{1}, \ldots, t_{k}\right)}\right]=\operatorname{Cov}\left[\mathbf{X}, v_{s}^{\left(t_{1}, \ldots, t_{k}\right)}\right] . } \\
&\left(\operatorname{Cov}\left[v_{s}^{\left(t_{1}, \ldots, t_{k}\right)}\right]+\sigma_{n}^{2} I\right)^{-1} v_{s}^{\left(t_{1}, \ldots, t_{k}\right)} \\
& \operatorname{Cov}\left[\mathbf{X} \mid v_{s}^{\left(t_{1}, \ldots, t_{k}\right)}\right]=K-\operatorname{Cov}\left[\mathbf{X}, v_{s}^{\left(t_{1}, \ldots, t_{k}\right)}\right] . \\
&\left(\operatorname{Cov}\left[v_{s}^{\left(t_{1}, \ldots, t_{k}\right)}\right]+\sigma_{n}^{2} I\right)^{-1} \operatorname{Cov}\left[v_{s}^{\left(t_{1}, \ldots, t_{k}\right)}, \mathbf{X}\right]
\end{aligned}
$$

With the covariance between an observation and a single point:

$$
\operatorname{Cov}\left[v_{s}^{(t)}, \mathbf{X}_{i}\right]=\sum_{j}^{\Lambda} w_{j}^{(t)} K_{i j}
$$

And the covariance between observations at times $t_{l}$ and $t_{m}$ :

$$
\operatorname{Cov}\left[v_{s}^{\left(t_{l}\right)}, v_{s}^{\left(t_{m}\right)}\right]=\sum_{i}^{\Lambda} \sum_{j}^{\Lambda} w_{i}^{\left(t_{l}\right)} w_{j}^{\left(t_{m}\right)} K_{i j}
$$




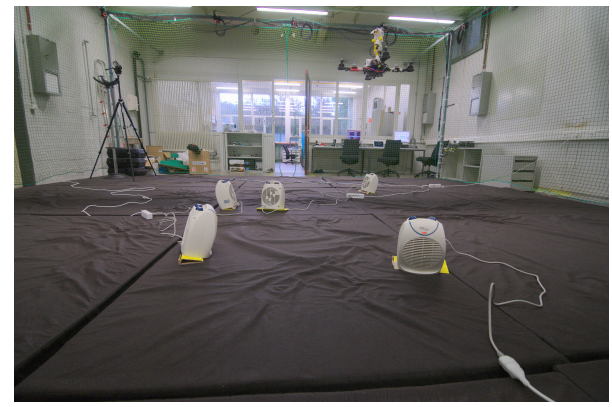

Fig. 4: Experiment in progress. Electrical heaters on the ground produce a surface temperature gradient to be estimated by the quadrotor.

Note that the posterior covariance (Equation 5) does not depend on the observed values, but only their statistics. This is a key feature of GPs, and it is of critical importance when it comes to path planning.

\section{Path Planning}

We implement and compare three approaches to guiding the quadrotor's sampling path: a naive "fixed grid" algorithm, which, given a period of time, evenly distributes its samples as a grid over the field, and two greedy algorithms that seek to maximize an information-theoretic quantity at each step.

1) Background: Our sampling strategies depend on two information theoretic quantities: entropy and mutual information.

Entropy can be described as a measure of the uncertainty about a random variable. For a given discrete random variable, $X$, its entropy is

$$
H(X)=-\sum_{i} P\left(X=x_{i}\right) \log \left(P\left(X=x_{i}\right)\right)
$$

We can further define the conditional entropy between two random variables to represent the the amount of uncertainty that remains once the other's value is known:

$$
H(X \mid Y)=\sum_{i, j} P\left(X=x_{i}, Y=y_{j}\right) \log \left(\frac{P\left(Y=y_{j}\right)}{P\left(X=x_{i}, Y=y_{j}\right)}\right)
$$

Mutual information is a measurement of the mutual dependence between two random variables. It can be defined in terms of entropy, as follows:

$$
\begin{aligned}
I(X ; Y) & =H(X)-H(X \mid Y) \\
& =H(Y)-H(Y \mid X)
\end{aligned}
$$

for two random variables $X$ and $Y$. Intuitively, mutual information is a measure of how much we learn about one variable, knowing the value of the other. If $X$ and $Y$ are independent, $I(X ; Y)=0$.

2) Sampling Strategies: Later in this paper we will build hybrid networks, where a mobile node operates in part with a static sensor network. Intelligently placing static nodes not only has a great effect on estimation quality, but may also determine the path of the quadrotor.
3) Static Sensor Sampling Strategy: Static sensor placement is well-studied, and has seen use in a number of applications. It is a key element of the design of an environmental sensing deployment. In the remainder of this paper, static nodes are always placed according to a greedy mutual information algorithm, as proposed in [3].

However, given that our sensors are not point sensors as in the above work, but measure an aggregate of the space inside their field of view (FOV), their full pose must be known in order to determine precisely what area of the space they are observing. We can then compute the mutual information as specified in [3], given that the covariance between two sensor observations in our model is given by Equation 7 .

4) Mobile Sensor Sampling Strategy: We consider two sampling strategies for the mobile node.

Fixed grid. Our naive approach simply distributes measurements evenly over the field, in a grid pattern. While simple, under our homogeneous GP assumption and for a fixed length of time, it is difficult to outperform.

Greedy information-theoretic. We propose two information-theoretic strategies, one that moves in the direction of maximum entropy, and another that moves in the direction that maximally increases information about the underlying GP.

Here we formulate the problem a bit differently than the static case above. Instead of an a priori fixed set of possible sampling locations as in [3], at each step we evaluate positions on the surface of a disk centered on the sensor's current position. The sensor moves in the direction that indicates the maximal increase in entropy or maximal information gain.

Formally, we first consider the mutual information case. The mobile sensor builds up a set of measured positions over the course of an experiment, $A$. At each step, we enumerate points on a disk centered on the sensor with radius determined by its maximum speed. Of these, we choose the point $y$ that maximally increases our information about the set of points in the underlying GP, $S$. That is, we seek to maximize $I(A \cup y ; S)-I(A ; S)$.

Again, in a similar fashion to [3], we can derive

$$
\begin{aligned}
I(A \cup y ; S)-I(A ; S) & \\
= & H(A \cup y)-H(A \cup y \mid S)-[H(A)-H(A \mid S)] \\
= & H(A \cup y)+H(S)-H(A \cup S \cup y) \\
& \quad-[H(A)+H(S)-H(A \cup S)] \\
= & H(A \cup y)-H(A)-[H(A \cup S \cup y)-H(A \cup S)] \\
= & H(y \mid A)-H(y \mid A \cup S)
\end{aligned}
$$

as our quantity to optimize. The maximum entropy strategy simply drops the second part of the equation, sampling to maximize $H(y \mid A)$.

\section{EXPERIMENTAL SETUP}

We validate our approach in a real-world setting with thermal gradients, generated by randomly distributing a set of electrical heating units in an indoor arena. Each heater is tilted downwards by approximately $10^{\circ}$ to increase the effect on the arena floor (see example of the setup in Figure 4). In this foundational work, we consider the resulting field to be a homogeneous GP. Nonetheless, both our estimation and 
our path planning algorithms are able to deal with spatially nonhomogeneous fields.

The arena is equipped with a MotionAnalysis Osprey motion capture system (MCS), comprised of 20 cameras [17]. The system provides real-time positioning at $100 \mathrm{~Hz}$ with millimeter precision, over a total volume of $3 \times 3 \times 2 \mathrm{~m}$ established for our experiments. The quadrotor flies through the arena using the absolute positioning provided by the MCS, streamed to the quadrotor over $\mathrm{Wi}-\mathrm{Fi}$, where it is fused with measurements from its inertial measurement unit (IMU). While flying, it measures the generated gradient with an onboard infrared thermometer facing downwards. Due to the limited space of our flying arena, and the narrow FOV of our mounted sensor, we performed all our scans at a fixed height of $1.8 \mathrm{~m}$.

A FLIR A320 thermal camera (see Figure 5) is used to precisely measure the thermal gradients generated by various placements of our electrical heaters. This surface temperature map is then used to characterize the field, as above, and to evaluate the performance of our sampling strategies.

Due to the camera's limited $25^{\circ} \mathrm{FOV}$, and the low ceiling in the experimental arena, it is not possible to take a picture of the whole field at once, and it must instead be constructed from a series of thermal images taken from different positions. Because the thermal camera's pose is known and the field of interest is flat, we are able to project the thermal image into the ground plane using simple geometry. The camera's true pose with respect to the MCS is found by using heated reflective markers on a frame (see Figure 5c). They allow us to find 2D-3D point correspondences between the thermal camera imagery and the pose estimated by the MCS, respectively. Examples of the resulting ground truth image can be seen in Figure 6.

Performance evaluation is then made possible by taking the RMSE of the quadrotor's field estimation with respect to a ground truth field generated as above. A set of ten fields were used. Their empirical covariance was computed, and we found them to be a good fit using the Gaussian covariance model with $a=0.646 \mathrm{~m}$. The resulting variogram can be seen in Figure 7. Again, given the assumptions outlined above, this function fully characterizes the spatial structure of the field.

The algorithms are also tested in a simulation environment, capable of generating well-behaved gradients. This allows us to validate the experiments conducted on the real setup.

\section{RESULTS}

We evaluate all three algorithms-fixed grid, greedy maximum entropy, and greedy mutual information-both in simulation and our indoor testbed. The simulation results use random fields generated according to the covariance function fitted to the ground truth fields observed in our testbed. In our real-world flights, each experimental run proceeds as follows. First, we place the heaters in the arena with random positions and headings. The ground truth temperature gradient is then acquired using the thermal camera. Finally, the quadrotor flies each generated path, while logging temperature and position

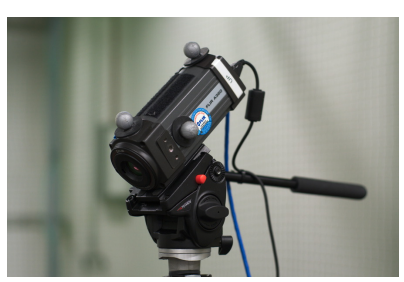

(a) FLIR A320 thermal camera with infrared reflectors for absolute positioning.

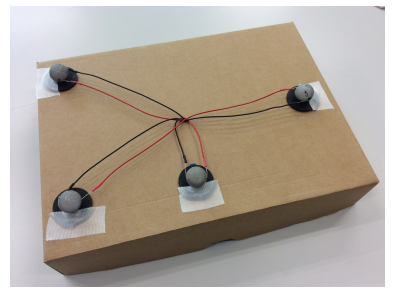

(c) Calibration frame with heated infrared markers.

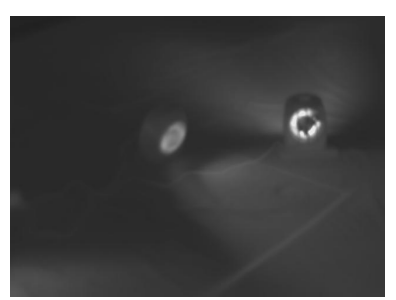

(b) Thermal image taken by the FLIR thermal camera during the ground truth construction process.

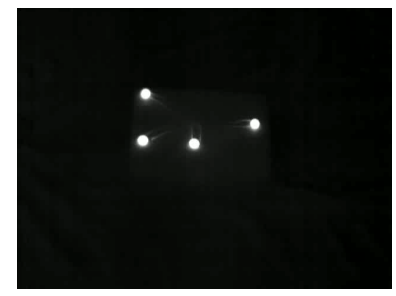

(d) Calibration frame as seen by the thermal camera.
Fig. 5: The thermal gradients produced by our electrical heaters are measured using a thermal camera. Each image taken by the camera is associated with its position and orientation, obtained by correcting MCS pose estimates with a corrective transform, obtained by calibrating the camera with a handmade frame.
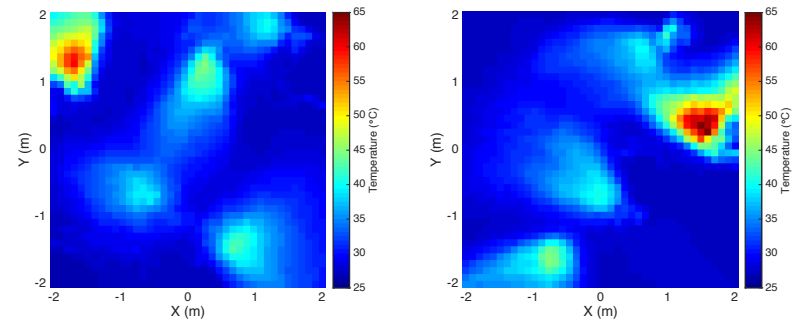

Fig. 6: Examples of ground truth surface temperature fields constructed using the thermal camera.

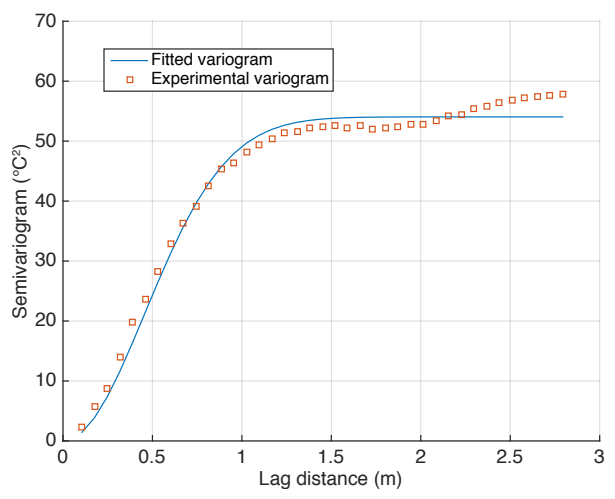

Fig. 7: Experimental and fitted variogram for a set of ten of our ground truth surface temperature maps, each representing an instantiation of our random electrical heater-based thermal gradients. The fitted covariance model is Gaussian, with $a=0.646 \mathrm{~m}$. 


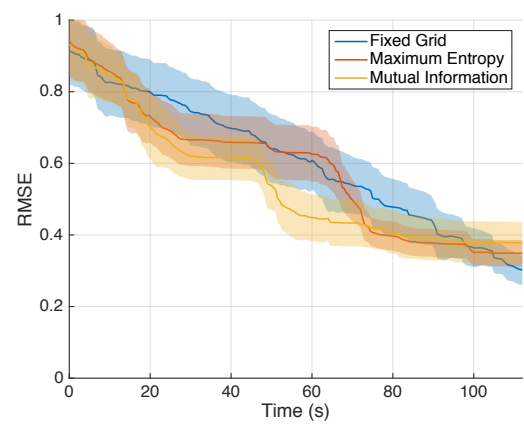

(a) Without static sensors.

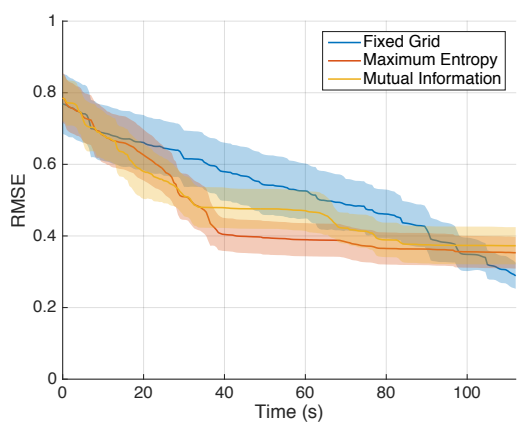

(b) Mobile sensor and ten static sensors.

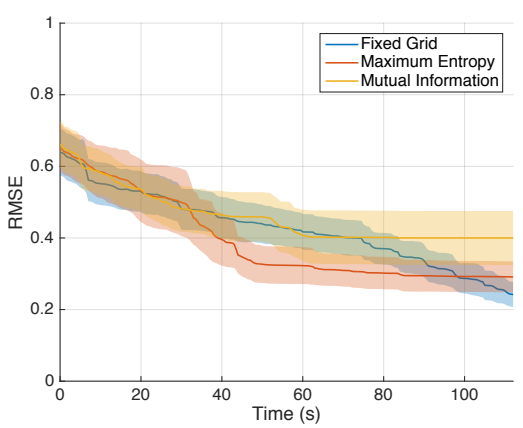

(c) Mobile sensor and twenty static sensors.

Fig. 8: Performance over the course of simulation for all three algorithms. Solid line represents the mean RMSE of the estimate, while the shaded area represents its standard deviation. Static sensors are placed before the start of the experiment (i.e., before time zero above).

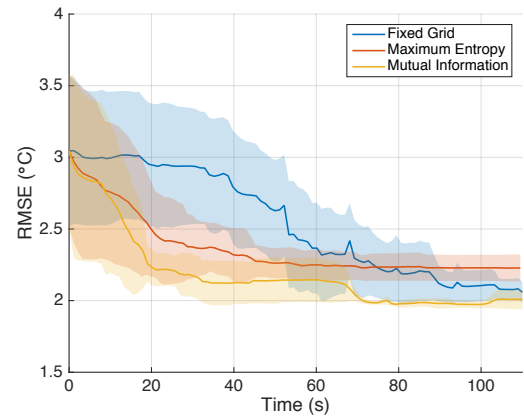

(a) Mobile sensor only.

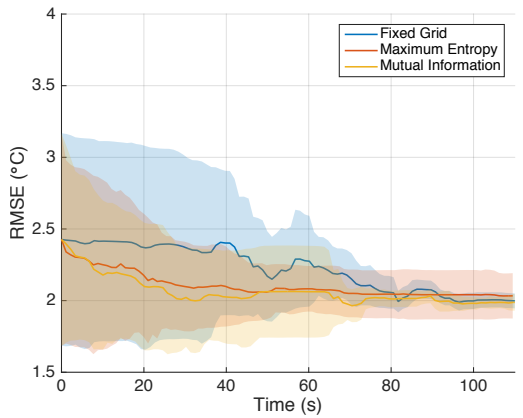

(b) Mobile sensor and ten static sensors.

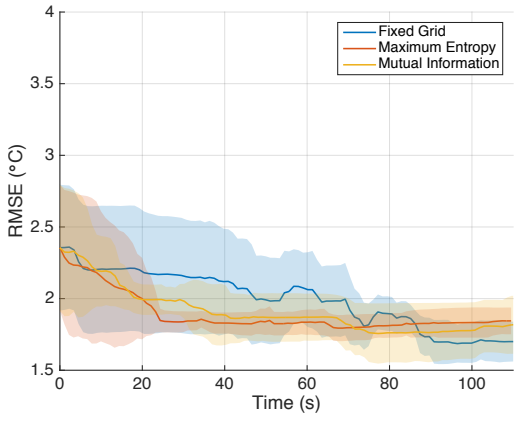

(c) Mobile sensor and twenty static sensors.

Fig. 9: Performance over the course of real-robot experiment for all three algorithms. Solid line represents the RMSE of the estimate, while the shaded area represents the standard deviation. Static sensors are placed before the start of the experiment.

data. Recall that for a given experimental configuration-i.e., field size, statistical structure, and static node placement- the path generated by all three of the above algorithms can be computed a priori.

For each algorithm, we present performance results for 1) a mobile sensor operating alone, 2) a mobile sensor operating over a field of ten simulated static nodes, and 3) over a field of twenty simulated static nodes. As described above, the static nodes are placed by a greedy mutual information strategy.

\section{A. Experiments}

We present simulation results in Figure 8. Each algorithm was evaluated over two hundred runs, and each run used a uniquely generated random field (Gaussian covariance model with $a=0.646 \mathrm{~m}$ ).

Five real-robot experimental runs were performed in our indoor testbed for each algorithm and for each possible sensor configuration. A comparison of the estimated root-meansquared error (RMSE) over the course of the each experiment is given in Figure 9.

Examples of real-robot trajectories and estimation results are given for a mobile sensor acting alone (Figure 10), a mobile sensor in conjunction with ten static nodes (Figure 11), and a mobile sensor in conjunction with twenty static nodes (Figure 12).

\section{B. Discussion}

First, we note a strong qualitative matching between our real-robot experiments and simulation results. In both simulation and reality, the greedy information-theoretic algorithms take an early lead, seeking out the most informative areas of the field, while the fixed grid approach, finishes with a higherquality estimate-note that due to our assumption of field homogeneity, the fixed grid approach optimally distributes the samples for a given time budget.

Also notable is that while the mutual information-based strategy performs almost strictly better than the maximum entropy strategy in an open arena, the latter is better able to find pockets of information in the field in the presence of previously deployed static nodes.

As the number of static sensors increases, note that the information-theoretic algorithms stay competitive with the fixed grid approach later into the experiments. Early in the estimation process, the fixed grid approach takes many measurements that are redundant with those taken by the static sensors. The adaptive algorithms are able to seek out more useful measurements by avoiding areas informed by the static 


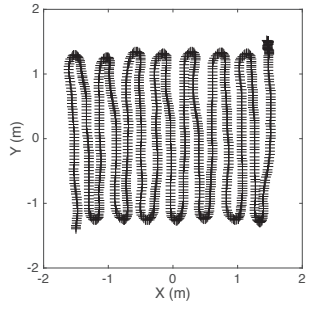

(a) Fixed grid path.

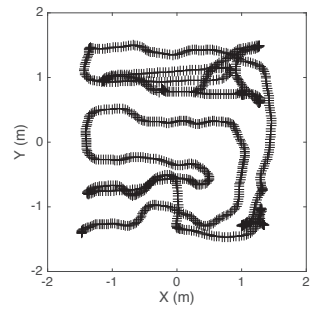

(c) Maximum entropy path.

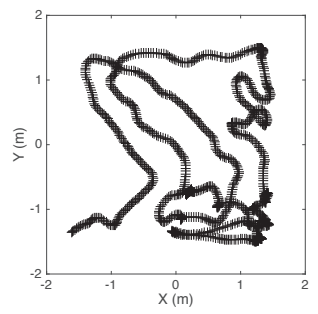

(e) Mutual information path.

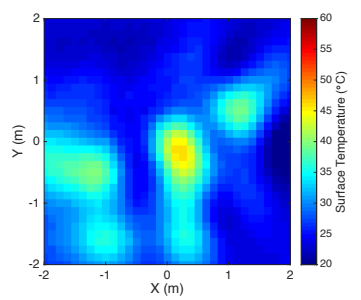

(b) Fixed grid posterior estimation.

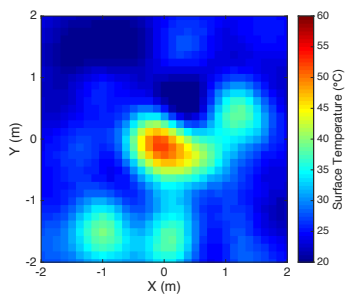

(d) Maximum entropy posterior estimation.

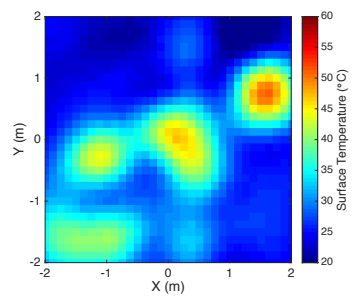

(f) Mutual information posterior estimation.
Fig. 10: Real-robot trajectories and example estimation results for a mobile sensor acting alone (i.e., without a supporting static network). (a), (c), (e) Actual trajectory of the quadrotor executing the fixed grid, maximum entropy, and mutual information strategies, respectively. (b), (d), (f) Example of final field estimate upon concluding each strategy.

sensors. This effect is magnified by increased static sensor density. Again, we observe this trend in both simulation and reality.

It is difficult however to quantitatively compare the simulations with real robot results. The real-robot experiments suffer many sources of error which are not explicitly modeled, and ultimately this leads to less accurate estimates than we observe in our simulation results. Such error sources include vibration of the sensor due to the quadrotor's flight, which may increase the area observed by the sensor; changes in the temperature of the sensor itself, which has a large influence on its measured surface temperature; or airflow from the rotors disrupting the thermal gradient on the floor of the arena, resulting in measurements that do not match the ground truth. Quantifying and otherwise addressing these issues will improve the overall estimation and should ultimately lead to results in line with the ideal case, as in our simulations.

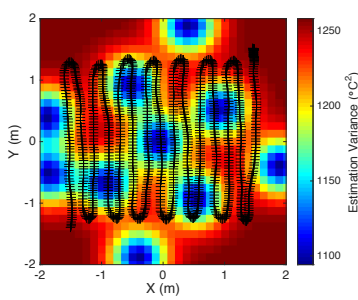

(a) Fixed grid path over static network posterior variance.

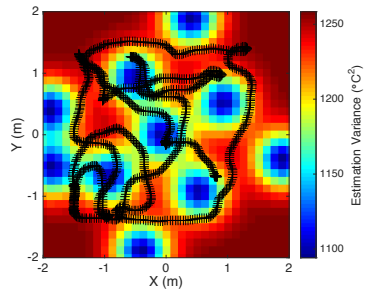

(c) Maximum entropy path over static network posterior variance.

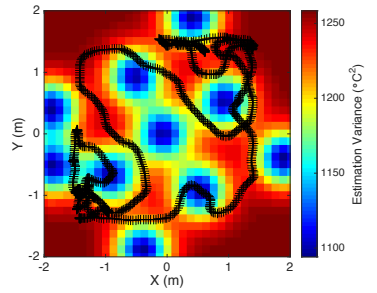

(e) Mutual information path over static network posterior variance.

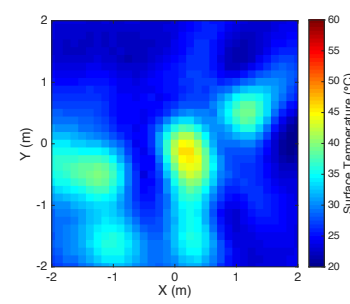

(b) Fixed grid posterior estimation.

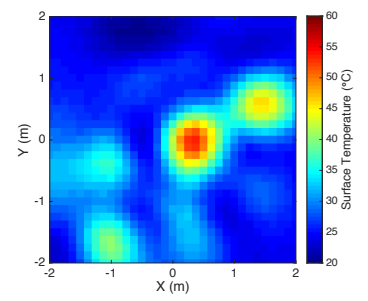

(d) Maximum entropy posterior estimation.

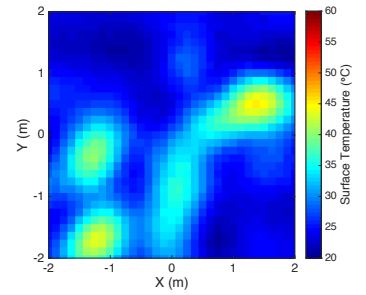

(f) Mutual information posterior estimation.
Fig. 11: Real-robot trajectories and example estimation results for a mobile sensor cooperating with a network of ten static nodes. (a), (c), (e) Actual trajectory of the quadrotor executing the fixed grid, maximum entropy, and mutual information strategies, respectively. Overlaid on the posterior estimation variance for the static network only. The blue low variance patches thus correspond to the ten static sensors placed. The two information-theoretic strategies tend to measure more frequently where the estimation variance is higher. (b), (d), (f) Example of final field estimate upon concluding each strategy.

\section{CONCLUSION}

Mobile and static sensor deployments are complementary to the monitoring of spatial environmental fields. In this work, we presented a comprehensive approach to estimating environmental fields using hybrid-mobility sensor networks. While we made strict assumptions on the statistical structure of the fields being estimated, we performed extensive validation of our approach in a novel semi-controlled real-world testbed. We believe this is a crucial intermediate step towards deploying more advanced algorithms in uncontrolled outdoor scenarios.

Many environmental fields have local nonstationarity-as is the case in our indoor testbed. While the general process by which we generate our thermal gradients can only be described as stationary, local anisotropies exist, for instance following 


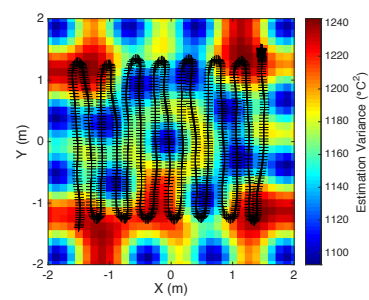

(a) Fixed grid path over static network posterior variance.

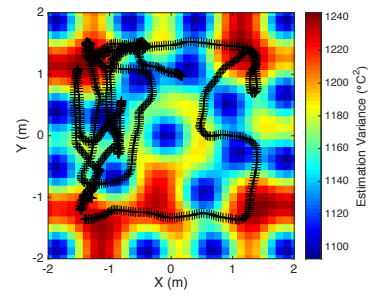

(c) Maximum entropy path over static network posterior variance.

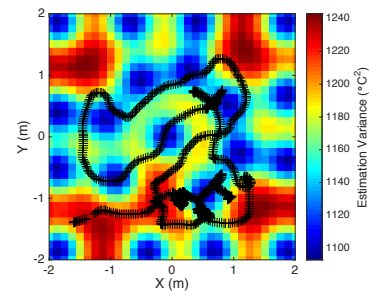

(e) Mutual information path over static network posterior variance.

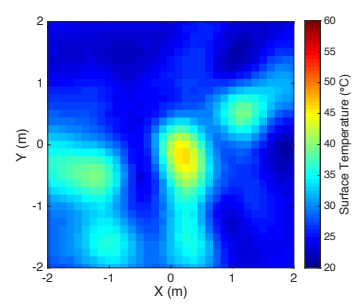

(b) Fixed grid posterior estimation.

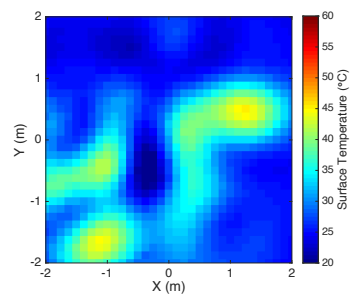

(d) Maximum entropy posterior estimation.

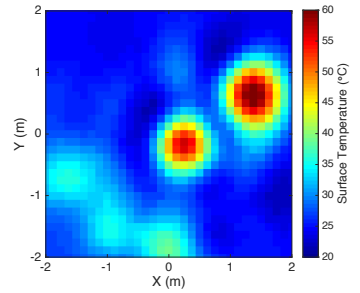

(f) Mutual information posterior estimation.
Fig. 12: Real-robot trajectories and example estimation results for a mobile sensor cooperating with a network of twenty static nodes. (a), (c), (e) Actual trajectory of the quadrotor executing the fixed grid, maximum entropy, and mutual information strategies, respectively. Overlaid on the posterior estimation variance for the static network only. The blue low variance patches thus correspond to the twenty static sensors placed. The two information-theoretic strategies tend to measure more frequently where the estimation variance is higher. (b), (d), (f) Example of final field estimate upon concluding each strategy.

the direction of a heat vent. Ouyang et al. have evaluated in simulation an approach to mobile sampling that is "piecewisestationary", i.e., they fit local stationary kernels to different parts of the field in an online fashion, as measurements are taken [7]. This line of approach is able to represent more complex spatial structures.

Due to the sparse distribution of heaters in our experiments, the testbed field often has large spaces with zero gradient. The presence of such areas makes it difficult to fit a single stationary model to the field. Interestingly, we note that this problem also appears in the study of rainfall prediction: large areas of the space may be dry, while areas experiencing nonzero rainfall are somehow well-related. Schleiss et al. propose a sort of masking technique, in which areas not experiencing the phenomenon are treated separately from those that are [18]. Integrating such an approach with a mobile sensor may further improve the results reported in this work.

\section{ACKNOWLEDGMENTS}

The authors would like to thank Prof. Paul Flikkema for inspirational discussion.

\section{REFERENCES}

[1] senseFly. Official website. [Online]. Available: https://www.sensefly. com/

[2] W. F. Caselton and J. V. Zidek., "Optimal monitoring network designs," Statistics and Probability Letters, vol. 2, no. 4, pp. 223-227, 1984.

[3] A. Krause, A. Singh, and C. Guestrin, "Near-optimal sensor placements in gaussian processes: Theory, efficient algorithms and empirical studies," Journal of Machine Learning Research, vol. 9, pp. 235-284, June 2008.

[4] B. J. Julian, M. Angermann, M. Schwager, and D. Rus, "Distributed robotic sensor networks: An information-theoretic approach," International Journal of Robotic Research, vol. 31, no. 10, pp. 1134-1154, 2012.

[5] N. Cao, K. H. Low, and J. M. Dolan, "Multi-robot informative path planning for active sensing of environmental phenomena: A tale of two algorithms," in Proceedings of the 2013 International Conference on Autonomous Agents and Multi-agent Systems, May 2013, pp. 7-14.

[6] G. Hoffmann and C. Tomlin, "Mobile sensor network control using mutual information methods and particle filters," IEEE Transactions on Automatic Control, vol. 55, no. 1, pp. 32-47, Jan. 2010.

[7] R. Ouyang, K. H. Low, J. Chen, and P. Jaillet, "Multi-robot active sensing of non-stationary gaussian process-based environmental phenomena," in Proceedings of the 2014 International Conference on Autonomous Agents and Multi-agent Systems, ser. AAMAS '14. Richland, SC: International Foundation for Autonomous Agents and Multiagent Systems, 2014, pp. 573-580.

[8] Ascending Technologies GmbH. Asctec hummingbird. [Online]. Available: http://www.asctec.de/en/uav-uas-drone-products/ asctec-hummingbird/

[9] ROS Wiki. asctec_mav_framework. [Online]. Available: http://wiki.ros. org/asctec_mav_framework

[10] M. Quigley, K. Conley, B. P. Gerkey, J. Faust, T. Foote, J. Leibs, R. Wheeler, and A. Y. Ng, "ROS: an open-source robot operating system," in ICRA Workshop on Open Source Software, May 2009.

[11] Phidgets, Inc. Phidgets 1045 infrared temperature sensor. [Online]. Available: http://www.phidgets.com/products.php?product_id=1045

[12] C. Huang, J. R. Townshend, S. Liang, S. N. Kalluri, and R. S. DeFries, "Impact of sensor's spread function on land cover characterization: assessment and deconvolution," Remote Sensing of Environment, vol. 80, pp. 203-212, 2002.

[13] Y. Akhtman, D. Constantin, M. Rehak, V. Nouchi, G. Shinkareva, D. Bouffard, N. Pasche, S. Chalov, U. Lemmin, and B. Merminod, "Leman-baikal: Remote sensing of lakes using an ultralight plane," in 6th Workshop on Hyperspectral Image and Signal Processing, Lausanne, Switzerland, 2014.

[14] C. C. D. Lelong, P. C. Pinet, and H. Poilvé, "Hyperspectral imaging and stress mapping in agriculture: A case study on wheat in Beauce (France)," Remote Sensing of Environment, vol. 66, no. 2, pp. 179-191, Nov. 1998.

[15] P. J. Zarco-Tejada, V. González-Dugo, and J. A. J. Berni, "Fluorescence, temperature and narrow-band indices acquired from a UAV platform for water stress detection using a micro-hyperspectral imager and a thermal camera," Remote Sensing of Environment, vol. 117, pp. 322-337, 2012, remote Sensing of Urban Environments.

[16] D. Stajnko, M. Lakota, and M. Hočevar, "Estimation of number and diameter of apple fruits in an orchard during the growing season by thermal imaging," Computers and Electronics in Agriculture, vol. 42, no. 1, pp. 31-42, 2004

[17] M. A. Corporation. Osprey digital realtime system. [Online]. Available: http://www.motionanalysis.com/pdf/Osprey_System.pdf

[18] M. Schleiss, S. Chamoun, and A. Berne, "Nonstationarity in intermittent rainfall: The 'dry drift'," Journal of Hydrometeorology, vol. 15, no. 3, pp. 1189-1204, 2014. 